\title{
Feedback Control on the Velocity Field and Source Term of a Normal Flow Equation
}

\author{
Angelo Alessandri, Patrizia Bagnerini, Mauro Gaggero, Anna Rossi
}

\begin{abstract}
The normal flow equation is a nonlinear partial differential equation that is quite popular in numerous research fields related to the so-called level set methods. Specifically, we have investigated the feedback control of such an equation by proposing two different regulators. The first approach consists in considering the velocity field of the equation as a control action; in such a case a simple proportional regulator is proved to be stable. In the second case, the control acts on the source term, and it relies on a Luenberger observer that provides an estimate of the norm of the gradient involved in the normal flow equation. Also this controller is proved to be stable by using Lyapunov arguments. Simulation results are presented to show the effectiveness of the proposed approaches.
\end{abstract}

\section{INTRODUCTION}

The interest for the normal flow (NF) partial differential equation (PDE), or NF equation for short, is motivated, among the others, by the success of the so called level set methods [1]. Such methods are used to describe the motion of fronts in two or three dimensions with a number of applications to fluid dynamics, image processing, material science, and many other fields [2]-[6]. In this paper we present two different regulators for systems described by the NF equation with a control action that may be either the speed of the velocity vector orthogonal to the front represented by the level sets of the unknown function or the source term of the NF equation. Stability results are presented in both cases together with successful simulation results that show the effectiveness of the proposed approaches.

The literature on the control of systems described by PDEs is extremely vast. If we restrict our attention to hyperbolic PDEs, as they are quite close to Hamilton-Jacobi equations like the NF equation, various approaches have been proposed to construct stabilizing closed-loop controllers and observers with stable estimation error, even possibly combined together in cascade for the purpose of output feedback. Such stability results are usually established by proving the contraction properties of the corresponding semigroup operators [7], [8] or by using classical Lyapunov tools [9]-[11]. Besides, the backstepping paradigm has become pretty popular starting with the pioneering work by Smyshlyaev and Krstic [12] (see also [13]-[19]).

Concerning state estimation for systems described by PDEs, many observers are proposed in the literature (see,

A. Alessandri, P. Bagnerini. and A. Rossi are with the University of Genoa (DIME), Via all'Opera Pia 15, I-16145 Genoa, Italy (emails: alessandri@dime.unige.it; bagnerini@dime.unige.it; rossi@dime.unige.it).

M. Gaggero is with the National Research Council of Italy, Via De Marini 6, I-16149 Genoa, Italy (email: mauro.gaggero@cnr.it).

The authors gratefully acknowledge financial support from the AFOSR with grant FA9550-15-1-0530. among others, [20]-[26]). Here, we rely on the use of a nonlinear Luenberger observer for the purpose of control (see also [27], [28]).

In this paper, we derive novel feedback controllers for systems described by a NF equation. In more detail, the control action may be either the speed coefficient of the velocity vector directed towards the normal to the front in all points or the source term of the equation. In the first case, a simple proportional controller is proposed, while in the second one a more complex scheme is developed that is based on a Luenberger observer providing an estimation of the norm of the gradient involved by the NF equation. Rigorous proofs of stability for the resulting controllers are derived using Lyapunov arguments [29]. The effectiveness of the proposed controllers is tested in different numerical examples, showing the pros and cons of the proposed approaches.

The paper is organized as follows. Section II reports the basic definitions that will be used in the following. The two proposed control schemes and the corresponding stability are presented in Section III. Section IV illustrates the simulation results, while conclusions are reported in Section V.

\section{PREliminaries}

The set of the nonnegative real numbers is denoted by $\mathbb{R}_{>0}$, while $\mathbb{R}_{>0}$ denotes the strictly positive real numbers. For any integer $n \geq 1$ and $x \in \mathbb{R}^{n}$, let $|x|:=\sqrt{\sum_{i=1}^{n} x_{i}^{2}}$.

Consider the Hamilton-Jacobi equation

$\phi_{t}(x, t)+H(x, \phi(x, t), \nabla \phi(x, t))=0,(x, t) \in \Omega \times \mathbb{R}_{\geq 0}$

where $\Omega \subset \mathbb{R}^{q}$ is compact, $H: \Omega \times \mathbb{R} \times \mathbb{R}^{q} \rightarrow \mathbb{R}$ is the Hamiltonian function, and let the bounded and uniformly continuous function $\phi(x, t)$ be a viscosity solution of (1) with initial condition $\phi(x, 0)=\phi_{0}(x) . \mathcal{L}_{2}(\Omega)$ denotes the Hilbert space of square integrable functions $\gamma: \Omega \rightarrow \mathbb{R}^{q}$ with norm $|\gamma|_{\mathcal{L}_{2}}=\left(\int_{\Omega}|\gamma(x, t)|^{2} d x\right)^{1 / 2}<\infty$ for all $t \geq 0$. $\mathcal{H}^{1}(\Omega)$ denotes the Sobolev space of square integrable functions with square integrable first derivatives, i.e., $\mathcal{H}^{1}(\Omega):=\left\{\gamma \in \mathcal{L}_{2}(\Omega): \nabla \gamma \in \mathcal{L}_{2}(\Omega)\right\}$. Finally, let $\varphi: \Omega \rightarrow$ $\mathbb{R}$ be an equilibrium of (1) with initial condition $\phi_{0}(x)$ if $H(x, \varphi(x), \nabla \varphi(x))=0$. Then, the solution $\phi(x, t) \in \mathcal{H}^{1}(\Omega)$ of (1) is said to be:

- $\mathcal{L}_{2}$ stable to $\varphi(x)$ if for all $\varepsilon>0$ there exists $\delta_{\varepsilon}>0$ such that

$$
\left|\phi_{0}-\varphi\right|_{\mathcal{L}_{2}}<\delta_{\varepsilon} \Rightarrow|\phi-\varphi|_{\mathcal{L}_{2}}<\varepsilon
$$

for all $t \geq 0$; 
- $\mathcal{L}_{2}$ asymptotically stable to $\varphi(x)$ if it is stable and

$$
\lim _{t \rightarrow+\infty}|\phi-\varphi|_{\mathcal{L}_{2}}=0 ;
$$

- $\mathcal{L}_{2}$ exponentially stable to $\varphi(x)$ if there exists $\lambda>0$ such that

$$
|\phi-\varphi|_{\mathcal{L}_{2}} \leq c\left|\phi_{0}-\varphi\right|_{\mathcal{L}_{2}} \exp (-\lambda t)
$$

for some $c>0$ and all $t \geq 0$.

In case

$$
|\phi-\varphi|_{\mathcal{L}_{2}} \leq c \exp (-\lambda t)
$$

holds instead of (2) (i.e., without explicit dependence on $\left|\phi_{0}-\varphi\right|_{\mathcal{L}_{2}}$ in the r.h.s.), we simply say that $\phi(x, t)$ converges exponentially to $\varphi(x)$ in $\mathcal{L}_{2}$ sense. Finally, the Young inequality is the upper bound of the cross product of any couple of real numbers $a$ and $b$ as follows: $2 a b \leq a^{2}+b^{2}$.

\section{CONTROL Schemes FOR THE NF EQUATION}

In this section, we present two control schemes for the NF equation. Generally speaking, first of all consider the $\mathrm{NF}$ equation

$$
\phi_{t}(x, t)+g(x, t)|\nabla \phi(x, t)|=h(x, t) \quad \text { in } \Omega \times[0,+\infty)
$$

where $g: \Omega \times[0,+\infty) \rightarrow \mathbb{R}$ and $h: \Omega \times[0,+\infty) \rightarrow \mathbb{R}$ are the velocity field and the source term, respectively. First, we will consider (3) with control input given by the velocity field. For the sake of brevity, we will refer to this case as "velocity field control," or VFC for short. Then, we will focus on (3) with control input represented by the source term, and we will call such a case as "source term control," or simply STC. In the following, we will investigate both approaches in detail, providing rigorous proofs of stability. For the sake of brevity, we will focus on the proofs of stability to zero though we may deal with tracking problems in general. Moreover, for the same reason from now on we refer to the one-dimensional case, i.e., with $\Omega=[a, b]$ with $a<b$.

\section{A. Control in the Velocity Field}

We focus on (3) with control input given by the velocity field $g(x, t)$ and source term $h(x, t)$ equal to zero. In other words, we consider the following equation:

$$
\phi_{t}(x, t)+u(x, t)\left|\phi_{x}(x, t)\right|=0
$$

where $u(x, t)$ denotes the control input. To stabilize (4), we propose to use a feedback regulator as follows:

$$
u(x, t)=k \phi(x, t)
$$

where $k>0$ is a given coefficient. Such a choice guarantees the stability of the closed loop system, as proved by the following theorem.

Theorem 1: System (4) subject to a proportional feedback law (5) with gain $k>0$ is $\mathcal{L}_{2}$ stable to zero.

Proof: Given the Lyapunov functional

$$
V(t)=\frac{1}{2} \int_{\Omega} \phi(x, t)^{2} d x,
$$

owing to (4) it is straightforward to get

$$
\dot{V}(t)=-k \int_{\Omega} \phi(x, t)^{2}\left|\phi_{x}(x, t)\right| d x \leq 0
$$

for all $\phi(x, t) \in \mathcal{L}_{2}(\Omega)$ and thus conclude on the $\mathcal{L}_{2}$ stability to zero with gain $k>0$ by means of standard Lyapunov arguments [29].

Note that in general the above theorem ensures only stability, but not asymptotic stability.

\section{B. Control in the Source Term}

We consider the NF equation (3) with a fixed velocity field $f(x, t)$ and a control input given by the source term. In other words, instead of (4) we focus on the following equation:

$$
\phi_{t}(x, t)+f(x, t)\left|\phi_{x}(x, t)\right|=u(x, t)
$$

where $f: \Omega \times[0,+\infty) \rightarrow \mathbb{R}$ is a known smooth, bounded function acting as velocity field. From now on we suppose that $f(x, t)>0$ for $x \in \Omega, t \in[0,+\infty)$. Such assumption guarantees a coercive Hamiltonian, which is a condition, among others, that is required to ensure the existence of solutions for (6).

If we had at disposal the knowledge of the gradient of $\phi(x, t)$, it would be easy to set up a regulator that stabilizes the system to zero. For example, we could choose $u(x, t)=$ $-k \phi(x, t)+f(x, t)\left|\phi_{x}(x, t)\right|$. In the absence of any knowledge on $\phi_{x}(x, t)$, we may construct a suitable observer-based control scheme. More specifically, in the following firstly we will focus on a Luenberger observer for the second term in the 1.h.s. of (6), i.e., $\eta(x, t):=f(x, t)\left|\phi_{x}(x, t)\right|$. Then, we will put such an observer in the loop with the scope of compensating $\eta(x, t)$ with a suitable estimate $\hat{\eta}(x, t)$ to impose a stabilizing feedback.

In order to estimate $\hat{\eta}(x, t):=f(x, t)\left|\hat{\phi}_{x}(x, t)\right|$, we rely on a Luenberger observer

$\hat{\phi}_{t}(x, t)+f(x, t)\left|\hat{\phi}_{x}(x, t)\right|+\ell(\hat{\phi}(x, t)-\phi(x, t))=u(x, t)$

where $\ell>0$ is the gain and $\hat{\phi}(x, t)$ is the state of the observer.

Theorem 2: Observer (7) for system (6) provides an estimation error $\tilde{\phi}(x, t):=\phi(x, t)-\hat{\phi}(x, t)$ that is $\mathcal{L}_{2}$ exponentially stable to zero if $\ell>0$ and $\tilde{\phi}(a, t)=\tilde{\phi}(b, t)$ for all $t \geq 0$.

Proof: The time derivative of the Lyapunov functional

$$
V(t)=\frac{1}{2} \int_{\Omega} \tilde{\phi}(x, t)^{2} d x
$$

is

$$
\begin{aligned}
\dot{V}(t) & =-\ell \int_{\Omega} \tilde{\phi}(x, t)^{2} d x+\int_{\Omega} f(x, t) \tilde{\phi}(x, t)\left[\left|\hat{\phi}_{x}(x, t)\right|\right. \\
& \left.-\left|\phi_{x}(x, t)\right|\right] d x .
\end{aligned}
$$

For the sake of brevity, let

$$
F_{\phi}(x, t):=f(x, t) \tilde{\phi}(x, t)\left[\left|\hat{\phi}_{x}(x, t)\right|-\left|\phi_{x}(x, t)\right|\right] .
$$


Clearly, if

$$
\int_{\Omega} F_{\phi}(x, t) d x \leq 0
$$

from (8) we get $\dot{V}(t) \leq-\ell V(t)$ and immediately conclude the proof. Toward this end, we note that

$$
\begin{aligned}
& \int_{\Omega} F_{\phi}(x, t) d x=\int_{\left\{x \in \Omega: \tilde{\phi}(x, t) \tilde{\phi}_{x}(x, t) \geq 0\right\}} F_{\phi}(x, t) d x \\
& +\int_{\left\{x \in \Omega: \phi(x, t) \tilde{\phi}_{x}(x, t)<0\right\}} F_{\phi}(x, t) d x .
\end{aligned}
$$

The first term in the r.h.s. of (9) can be easily bounded by zero since $f(x, t)$ is smooth and bounded. Concerning the second term, the derivation of the same bound can be obtained by using the assumption that $f(x, t)$ is non negative. For the sake of space limitation, this proof is omitted.

Note that the condition $\tilde{\phi}(a, t)=\tilde{\phi}(b, t)$ can be satisfied by choosing $\phi(x, t)=\hat{\phi}(x, t)$ on the boundary.

Based on the estimate $\hat{\eta}(x, t):=f(x, t)\left|\hat{\phi}_{x}(x, t)\right|$, we can generate the control action

$$
u(x, t)=-\ell \phi(x, t)+(\ell-k) \hat{\phi}(x, t)+f(x, t)\left|\hat{\phi}_{x}(x, t)\right|
$$

in such a way to stabilize the system, as follows.

Theorem 3: The state of system (6) subject to (10) with $k>0, \ell>0$, and $\tilde{\phi}(a, t)=\tilde{\phi}(b, t)$ converges exponentially to zero in the $\mathcal{L}_{2}$ sense.

Proof: If we replace (10) in (7), we get $\hat{\phi}_{t}(x, t)=$ $-k \hat{\phi}(x, t)$ and hence, using the Lyapunov functional $V(t)=$ $\int_{\Omega} \hat{\phi}(x, t)^{2} d x / 2$, it is straightforward to prove the $\mathcal{L}_{2}$ exponentially stability of $\hat{\phi}(x, t)$ to zero. Since from the Young inequalities it follows

$$
\begin{aligned}
& \phi(x, t)^{2}=(\overbrace{\phi(x, t)-\hat{\phi}(x, t)}^{\tilde{\phi}(x, t)}+\hat{\phi}(x, t))^{2} \\
& \leq 2 \tilde{\phi}(x, t)^{2}+2 \hat{\phi}(x, t)^{2},
\end{aligned}
$$

we conclude on the $\mathcal{L}_{2}$ exponential convergence of $\phi(x, t)$ to zero owing to the $\mathcal{L}_{2}$ exponentially stability of both $\hat{\phi}(x, t)$ and $\tilde{\phi}(x, t)$ (from Theorem 2).

It is noteworthy that the special choice of $k$ just equal to $\ell$ provides the simple observer-based law

$$
u(x, t)=-k \phi(x, t)+f(x, t)\left|\hat{\phi}_{x}(x, t)\right|
$$

In the next section, we will analyze the effectiveness of the proposed control schemes by means of simulations.

\section{Simulation Results}

This section is focused on the numerical results we obtained in applying the proposed approaches to force a front to become another given reference front. As compared with previous results on the optimal control of moving fronts associated with the level set of a NF equation [30]-[32], the new results are obtained by using much simpler controllers.
A moving front is described by the zero level set of a certain function $\phi_{d}(x) \in \mathcal{H}^{1}(\Omega)$. Specifically, the zero level set of the function $\phi$ is given by the set-valued mapping $\Gamma:[0, T] \rightrightarrows \mathcal{C}$, where $\Gamma(t):=\{x \in \Omega: \phi(x, t)=0\}$. We will construct regulators for (3) such that $\Gamma(t)$ tracks the reference front $\Gamma_{d}:=\left\{x \in \Omega: \phi_{d}(x)=0\right\}$. Indeed, the proposed approaches will allow to track the entire function $\phi$ and not only its zero level set. As a consequence, all the level sets of $\phi$ will converge to the corresponding level sets of $\phi_{d}$.

We focus on case studies involving VFC and STC problems with a two-dimensional NF equation and different shapes of the reference curve given by the zero level set of the function $\phi_{d}(x)$. More specifically, we considered a circle, two ellipses, and a star-shaped curve, denoted as "Case A", "Case B", and "Case C", respectively. In all the examples, we fixed $f(x, t)=1$ for the STC.

In all the cases, the NF equations (4) or (6) were solved on a spatial domain $\Omega=[-0.5,+0.5] \times[-0.75,+0.75]$, discretized by using a regular grid of $50 \times 75$ points. Concerning the VFC approach, we fixed a time interval $[0,1.5]$, discretized with sampling time $\Delta t$ equal to 0.03 , i.e., 50 time steps were needed to complete the simulation. As regards the STC, we considered a time interval $[0,0.6]$ sampled with a total of 300 steps.

All the simulations were performed in Matlab on a personal computer with a $2.6 \mathrm{GHz}$ Intel Xeon CPU with $64 \mathrm{~GB}$ of RAM. In more detail, the Matlab toolbox implemented by Mitchell [33] was used. An upwind second-order essentially non-oscillatory scheme [6, chap. 3] with respect to the space was used for the numerical solution of the NF equations. Concerning the time approximation, we adopted a total variation diminishing Runge-Kutta scheme of second order.

Fig. 1 reports the snapshots of the fronts $\Gamma(t)$ and $\Gamma_{d}$ for the VFC approach. In more detail, the results of the Cases A and $\mathrm{C}$ were obtained with $k=20$, while the plots of Case B refers to $k=10000$. Fig. 2 sketches the snapshots of the fronts $\Gamma(t)$ and $\Gamma_{d}$ for the STC approach. Specifically, the results of the Cases $\mathrm{A}$ and $\mathrm{C}$ were obtained with $k=\ell=20$, while the plots of Case B refers to $k=\ell=1000$. In both the VFC and STC, the largest coefficient in the Case B is required by the intrinsic difficulty of this example, which involves a change of topology.

To evaluate the performances, we introduce the quantity $e(t)$, defined as the symmetric difference between the actual front $\Gamma(t)$ and the reference one $\Gamma_{d}$, i.e.,

$$
e(t)=\int_{\Omega} \Gamma(t) \Delta \Gamma_{d} d x
$$

where $\Delta$ is the symmetric difference operator, i.e., $A \Delta B=$ $(A \cup B) \backslash(A \cap B)$. Figs. 3 and 4 show the time behavior of $e(t)$ for the VFC and STC schemes, respectively.

It turns out that both the VFC and the STC are able to guarantee convergence to the reference front $\Gamma_{d}$ for all the considered shapes. The convergence speed of the VFC is higher than that of the STC, as it is evident by checking Fig.s 3 and 4. In general, the STC requires larger values for 

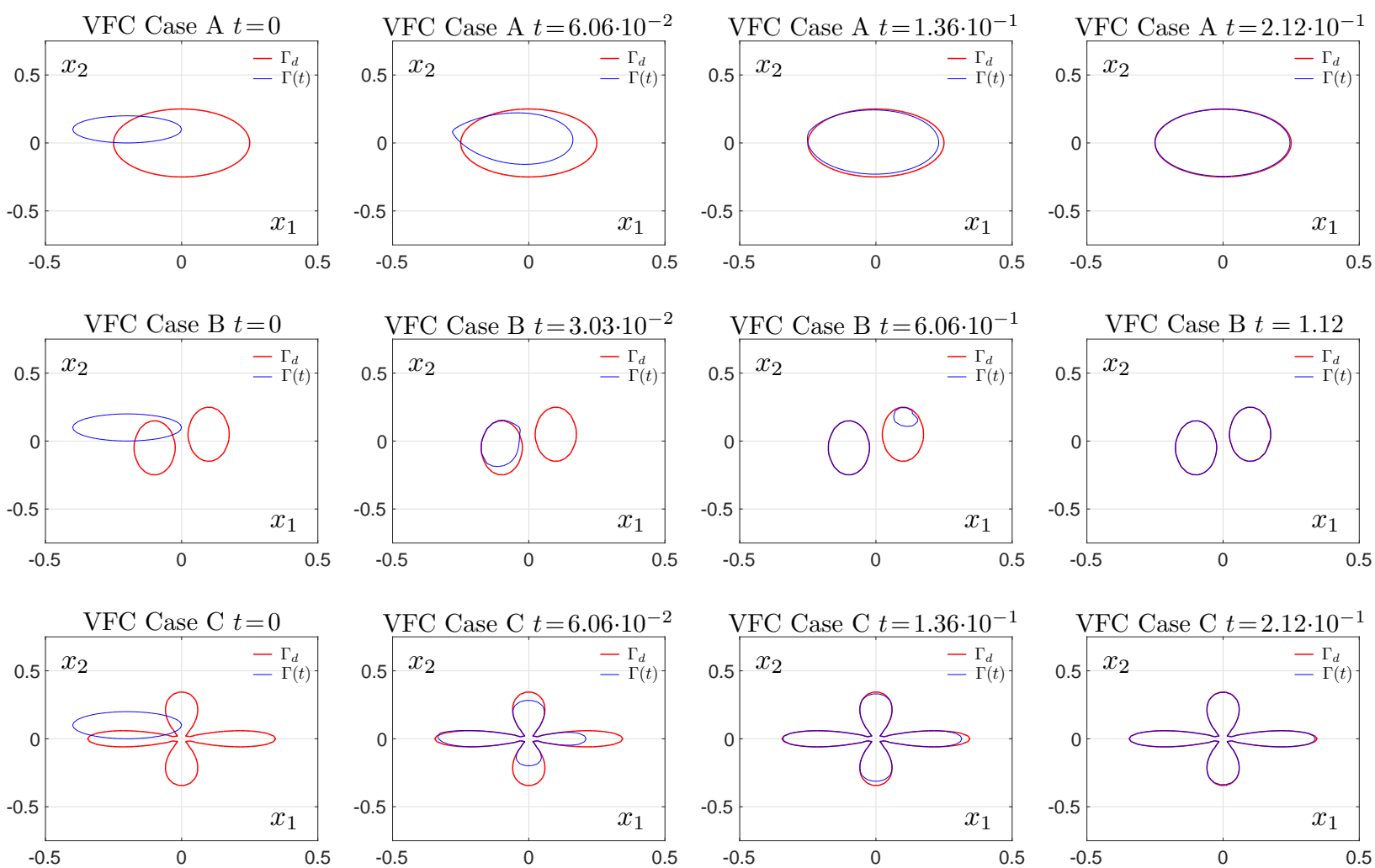

Fig. 1. Front tracking snapshots obtained with the VFC approach in the three considered cases.
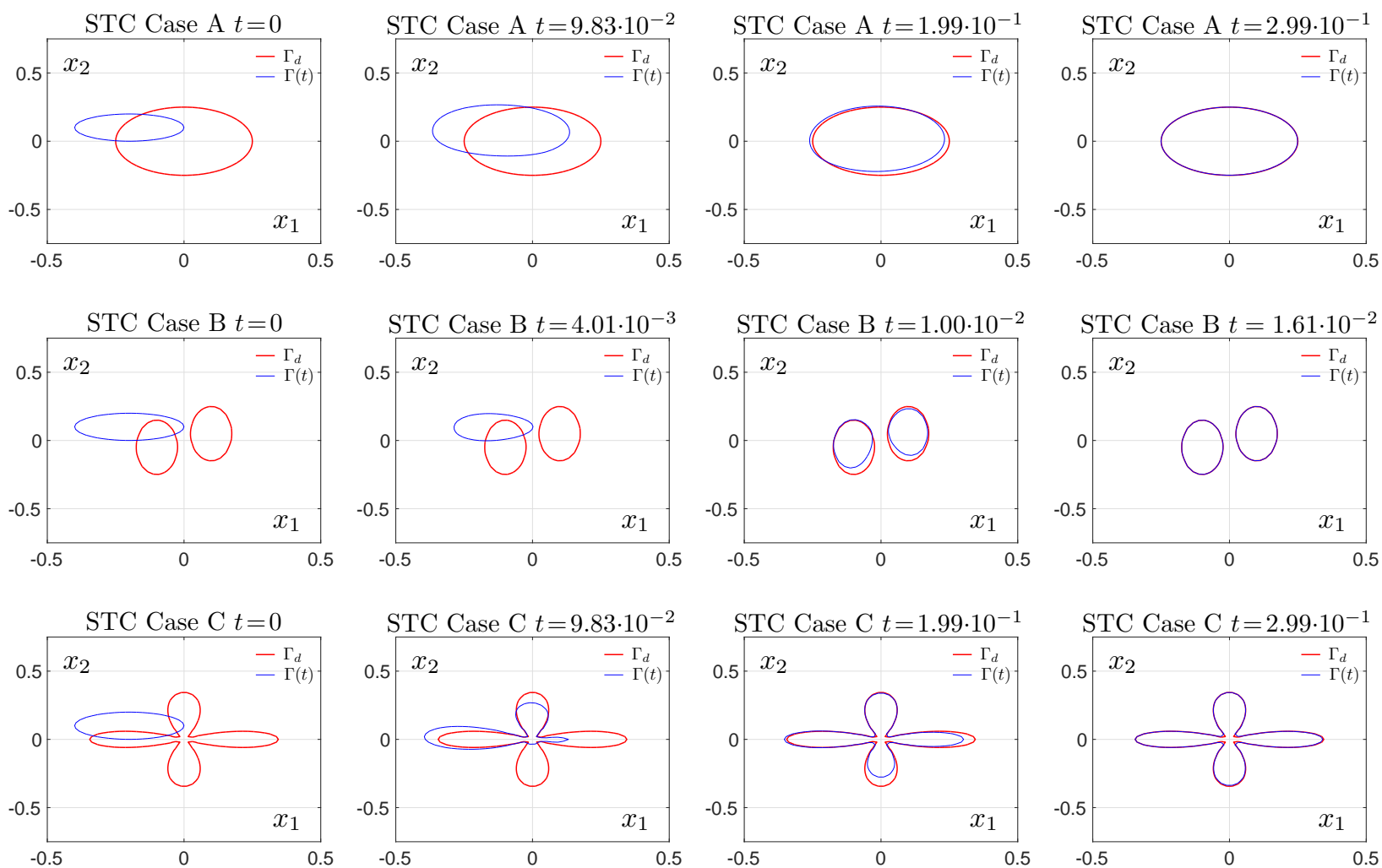

Fig. 2. Front tracking snapshots obtained with the STC approach in the three considered cases.

the parameter $k$ to obtain convergence with respect to the VFC. Such a behavior is ascribed to the the effect of the observer in the loop of the STC, whereas no estimator is required to control the fronts with the VFC. 

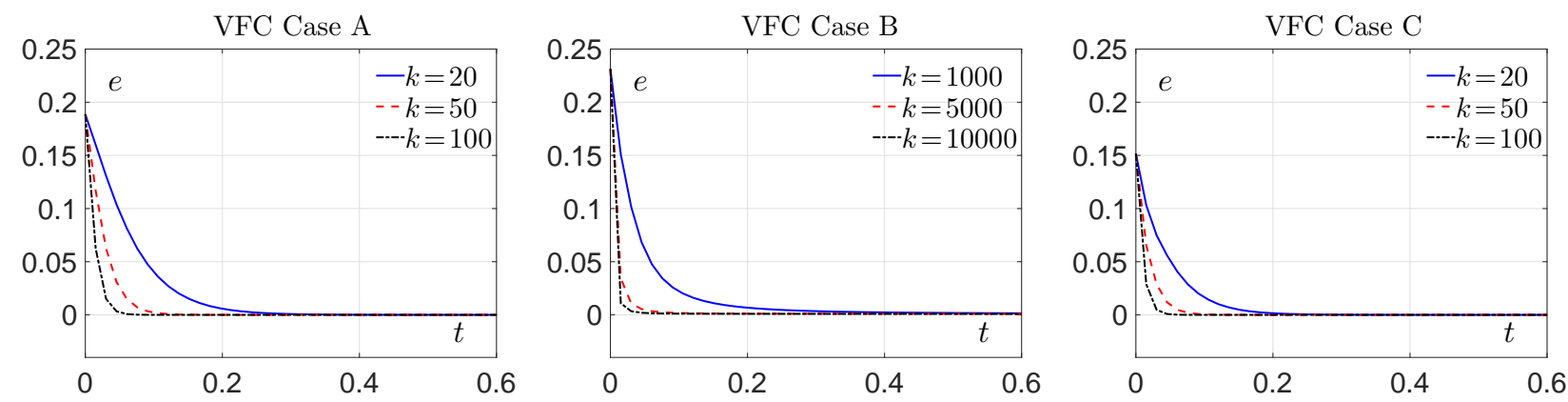

Fig. 3. Time decrease of the error $e$ for the VFC approach.
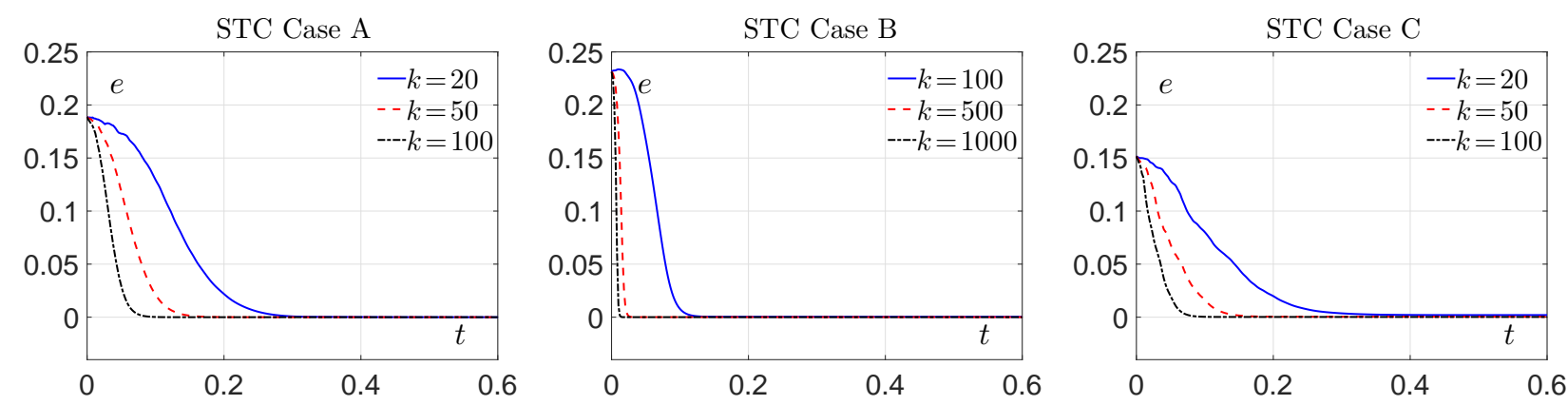

Fig. 4. Time decrease of the error $e$ for the STC approach.

As said, both the VFC and STC methods ensure the convergence of the entire function $\phi$ and not only its zero level set. As a consequence, all the level sets of $\phi$ converge to the corresponding level sets of $\phi_{d}$. Fig.s 5 and 6 confirm this, as they display the snapshots of the functions $\phi(x, t)$ at certain time steps and $\phi_{d}(x)$ for the VFC and STC approaches, respectively, in the first line and the corresponding level sets in the second one for the Case C. Similar results could be shown for the Cases A and B, but they are not reported for the sake of brevity.

\section{Conclusions}

In this paper, we have studied the feedback control of the NF equation by proposing two different regulators. In the first case, the control acts on the velocity field of the equation, and it is simply proportional. Moreover, it has been proved to be $\mathcal{L}_{2}$ stable. In the second case, the control action is in the source term and relies on a structure containing a Luenberger observer that provides an estimation of the norm of the gradient of $\phi(x, t)$ appearing in the NF equation. Successful simulation results with different shapes given by the zero level sets of the reference functions have been presented that confirm the theoretical findings. The existence of the solution of closed-loop NF equations is still an open problem that we are currently investigating.

\section{REFERENCES}

[1] S. Osher and J. Sethian, "Fronts propagating with curvature-dependent speed: algorithms based on Hamilton-Jacobi formulations," J. Comput. Phys., vol. 79, no. 1, pp. 12-49, 1988.

[2] J. Sethian, Level Set Methods and Fast Marching Methods, 2nd ed. Cambridge: Cambridge University Press, 1999, vol. 3.
[3] J. Berg and K. Holmström, "On parameter estimation using level sets," SIAM J. Control Optim., vol. 37, no. 5, pp. 1372-1393, 1999.

[4] J. Sethian and P. Smereka, "Level set methods for fluid interfaces," Annual Rev. Fluid Mechanics, vol. 35, no. 1, pp. 341-372, 2003.

[5] S. Osher and R. Fedkiw, Level set methods and dynamic implicit surfaces, ser. Applied Mathematical Sciences. New York: SpringerVerlag, 2003, vol. 153.

[6] R. Kimmel, Numerical Geometry of Images. New York: SpringerVerlag, 2004.

[7] A. A. Moghadam, I. Aksikas, S. Dubljevic, and J. F. Forbes, "Boundary optimal (LQ) control of coupled hyperbolic PDEs and ODEs," Automatica, vol. 49, no. 2, pp. 526 - 533, 2013.

[8] I. Aksikas, "Asymptotic behaviour of contraction non-autonomous semi-flows in a Banach space: Application to first-order hyperbolic PDEs," Automatica, vol. 65, pp. 140 - 146, 2016.

[9] M. Diagne, P. Shang, and Z. Wang, "Feedback stabilization for the mass balance equations of an extrusion process," IEEE Trans. on Automatic Control, vol. 61, no. 3, pp. 760-765, 2016.

[10] M. Ahmadi, G. Valmorbida, and A. Papachristodoulou, "Dissipation inequalities for the analysis of a class of PDEs," Automatica, vol. 66, pp. 163 - 171, 2016.

[11] M. Herty and W.-A. Yong, "Feedback boundary control of linear hyperbolic systems with relaxation," Automatica, vol. 69, pp. 12 $17,2016$.

[12] A. Smyshlyaev and M. Krstic, Boundary control of PDEs: A course on backstepping designs. SIAM, 2008, vol. 16.

[13] M. L. Marca, D. Armbruster, M. Herty, and C. Ringhofer, "Control of continuum models of production systems," IEEE Trans. on Automatic Control, vol. 55, no. 11, pp. 2511-2526, 2010.

[14] O. M. Aamo, "Disturbance rejection in $2 \times 2$ linear hyperbolic systems," IEEE Trans. on Automatic Control, vol. 58, no. 5, pp. 10951106, 2013.

[15] F. Bribiesca-Argomedo and M. Krstic, "Backstepping-forwarding control and observation for hyperbolic PDEs with Fredholm integrals," IEEE Trans. on Automatic Control, vol. 60, no. 8, pp. 2145-2160, 2015.

[16] H. Anfinsen and O. M. Aamo, "Disturbance rejection in the interior domain of linear $2 \times 2$ hyperbolic systems," IEEE Trans. on Automatic Control, vol. 60, no. 1, pp. 186-191, 2015.

[17] H. Anfinsen, M. Diagne, O. M. Aamo, and M. Krstic, "An adaptive observer design for $n+1$ coupled linear hyperbolic PDEs based on 

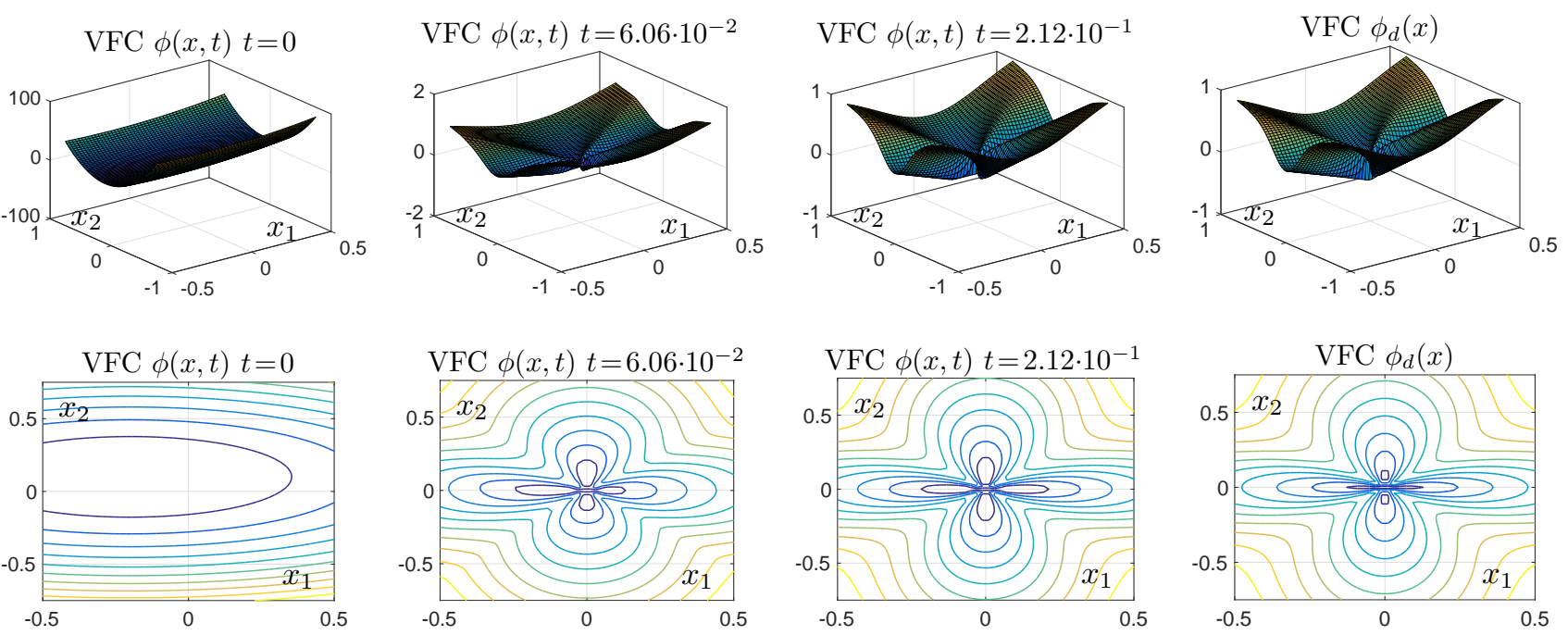

Fig. 5. Snapshots of the functions $\phi(x, t)$ and $\phi_{d}(x)$ (first line) and corresponding level sets (second line) for the VFC approach in the Case C.
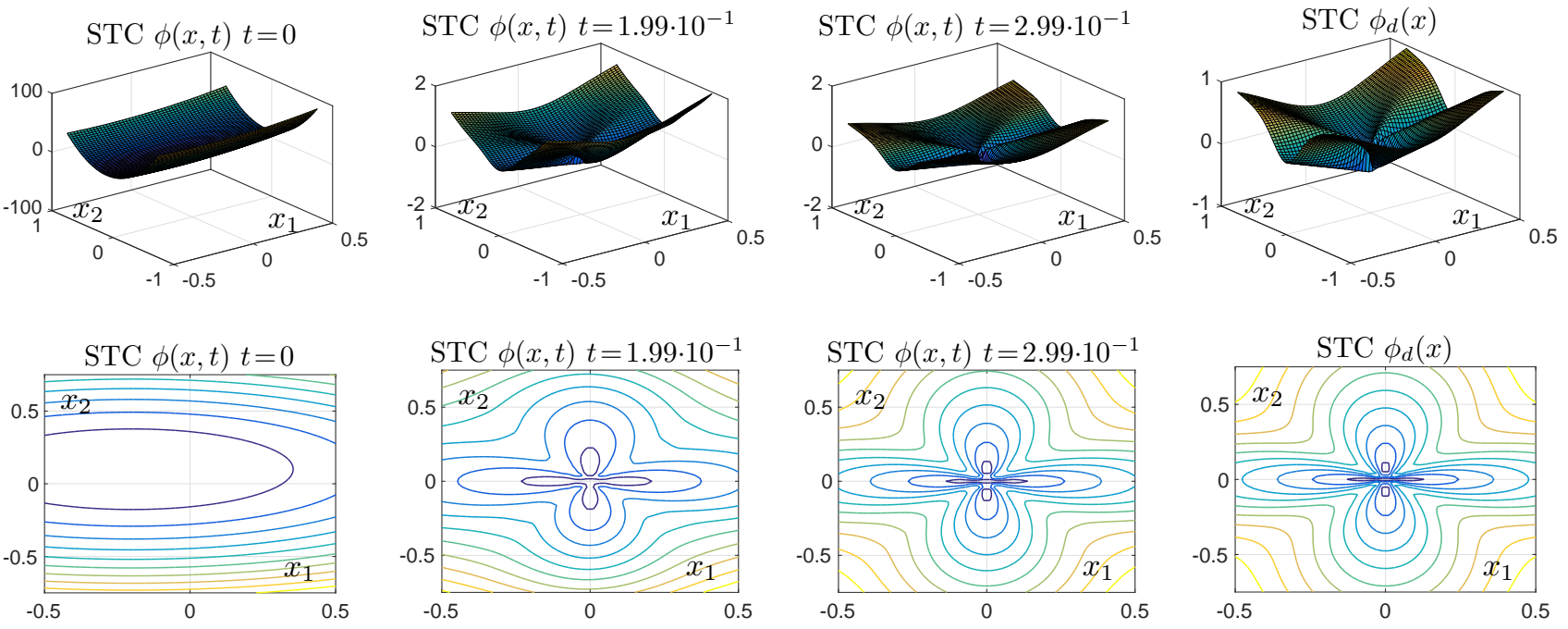

Fig. 6. Snapshots of the functions $\phi(x, t)$ and $\phi_{d}(x)$ (first line) and corresponding level sets (second line) for the STC approach in the Case C.

swapping," IEEE Trans. on Automatic Control, vol. 61, no. 12, pp. 3979-3990, 2016.

[18] L. Hu, F. D. Meglio, R. Vazquez, and M. Krstic, "Control of homodirectional and general heterodirectional linear coupled hyperbolic PDEs," IEEE Trans. on Automatic Control, vol. 61, no. 11, pp. 33013314,2016

[19] J. Deutscher, "Finite-time output regulation for linear $2 \times 2$ hyperbolic systems using backstepping," Automatica, vol. 75, pp. 54 - 62, 2017.

[20] W. Yu, "Identification for parabolic distributed parameter systems with constraints on the parameters and the state," SIAM Journal on Control and Optimization, vol. 33, no. 6, pp. 1801-1815, 1995.

[21] A. Smyshlyaev and M. Krstic, "On control design for pdes with space-dependent diffusivity or time-dependent reactivity," Automatica, vol. 41, no. 9, pp. 1601-1608, 2005.

[22] M. Efe, H. Ozbay, and M. Samimy, "Infinite dimensional and reduced order observers for Burgers equation," Int. Journal of Control, vol. 78, no. 11, pp. 864-874, 2005.

[23] D. Vries, K. Keesman, and H. Zwart, "Luenberger boundary observer synthesis for Sturm-Liouville systems," Int. Journal of Control, vol. 83, no. 7, pp. 1504-1514, 2010.

[24] S. Tang and C. Xie, "State and output feedback boundary control for a coupled PDE-ODE system," Systems \& Control Letters, vol. 60, no. 8, pp. 540-545, 2011.

[25] X.-D. Li and C.-Z. Xu, "Infinite-dimensional Luenberger-like ob- servers for a rotating body-beam system," Systems \& Control Letters, vol. 60, no. 2, pp. 138-145, 2011.

[26] T. Ahmed-Ali, F. Giri, M. Krstic, and F. Lamnabhi-Lagarrigue, "Observer design for a class of nonlinear ODE-PDE cascade systems," Systems \& Control Letters, vol. 83, pp. 19-27, 2015.

[27] M. Demetriou, "Design of consensus and adaptive consensus filters for distributed parameter systems," Automatica, vol. 46, no. 2, pp. 300-311, 2010.

[28] M. Demetriou, "Guidance of mobile actuator-plus-sensor networks for improved control and estimation of distributed parameter systems," IEEE Trans. on Automatic Control, vol. 55, no. 7, pp. 1570-1584, 2010.

[29] F. Mazenc and C. Prieur, "Strict Lyapunov functions for semilinear parabolic partial differential equations," Mathematical Control and Related Fields, vol. 1, no. 2, pp. 231-250, 2011.

[30] A. Alessandri, P. Bagnerini, and M. Gaggero, "Optimal control of level sets dynamics," in Proc. American Control Conference, 2014, pp. 2208-2213.

[31] A. Alessandri, P. Bagnerini, M. Gaggero, and A. Traverso, "Further results on the optimal control of fronts generated by level set methods," in Proc. American Control Conference, 2016, pp. 5225-5230.

[32] A. Alessandri, P. Bagnerini, and M. Gaggero, "Extended Kalman filtering to design optimal controllers of fronts generated by level set methods," in Proc. IEEE Conf. on Decision and Control, 2016, pp. 3966-3971. 
[33] I. Mitchell, "The flexible, extensible and efficient toolbox of level set methods," J. Scientific Comput., vol. 35, no. 2-3, pp. 300-329, 2008. 